Carnets de géographes

GÉOGRAPHES.

\title{
Dessiner les « fair trade towns »?
}

Réflexions sur le recours à la représentation graphique pour exprimer des territorialités d'acteurs

\section{Lisa Rolland}

\section{Q OpenEdition}

1 Journals

Édition électronique

URL : http://journals.openedition.org/cdg/320

DOI : $10.4000 /$ cdg. 320

ISSN : 2107-7266

Éditeur

UMR 245 - CESSMA

Référence électronique

Lisa Rolland, «Dessiner les « fair trade towns »? », Carnets de géographes [En ligne], 8 | 2015, mis en ligne le 01 septembre 2015, consulté le 20 avril 2019. URL : http://journals.openedition.org/cdg/320 ; DOI : $10.4000 / c d g .320$

Ce document a été généré automatiquement le 20 avril 2019

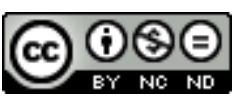

La revue Carnets de géographes est mise à disposition selon les termes de la Licence Creative Commons Attribution - Pas d'Utilisation Commerciale - Pas de Modification 4.0 International. 


\title{
Dessiner les « fair trade towns »?
}

\author{
Réflexions sur le recours à la représentation graphique pour exprimer \\ des territorialités d'acteurs
}

\section{Lisa Rolland}

\section{Introduction}

1 Un décentrement du commerce équitable vers d'autres espaces et dynamiques d'acteurs est perceptible autour de nouveaux enjeux liés à son inscription dans les politiques publiques (Smith, 2011). Cette évolution ouvre des perspectives originales de recherche et élargit le champ des disciplines concernées, avec notamment la géographie. Mon sujet de thèse, « Les"Fair Trade Towns", une mise en territoires du commerce équitable au nom du développement durable? Regard multi-sites France, Belgique", s'inscrit dans ces nouvelles approches: il questionne les espaces et les échelles du commerce équitable dans les" Fair Trade Towns". Cette campagne internationale de promotion du secteur, lancée en 2000 en Angleterre, prend place aujourd'hui dans 24 pays. Elle labellise des collectivités, essentiellement des villes européennes (1.350 sur un total de 1.431, selon le site des Fair Trade Towns) sur la base de critères homogénéisés. En principe, il y a cinq critères à respecter dans l'ordre pour être titré «Fair Trade Town»: adopter une résolution du conseil municipal et acheter des produits équitables, développer les produits dans les commerces et restaurants du territoire, inciter les entreprises du territoire à en acheter, communiquer sur les réalisations et sensibiliser, créer un conseil local, lieu d'échanges et de propositions (qui dans la pratique n'existe pas ou peu). L'objectif affiché est d'associer collectivités locales, acteurs locaux privés et publics, dans une démarche de promotion du commerce équitable à l'échelle d'un territoire centré autour de la ville. Les terrains d'étude sont situés à Lyon (France), qui a reçu le titre" Territoire de commerce équitable" depuis 2009, et Bruxelles-Capitale (Belgique)," Commune de commerce équitable" et" Fair Trade Gemeente" depuis 2004. En Belgique, la campagne prend deux formes différentes eu égard du découpage politique du pays, ce qui questionne le projet territorial de la campagne. En Wallonie, c'est le titre de «Communes de commerce équitable » qui est décerné et en Flandre celui de « Fair Trade Gemeente ». 
Bruxelles-Capitale est située en région Flandre mais dépend de la région WallonieBruxelles, elle a obtenu le titre dans les deux langues. Dans le cadre d'une réflexion plus large portant sur la structuration du territoire dans les discours et les pratiques des acteurs, cet article s'attache aux territorialités des acteurs, c'est-à-dire la dimension relationnelle du territoire (jeux d'acteurs, intérêts, contradictions).

2 La littérature scientifique sur le commerce équitable se caractérise par un déficit de mention de l'espace et de sa représentation. Ce manque de données géographiques interroge dans le sens où le commerce équitable renvoie à une conception du monde et du développement fondée sur la notion de solidarité. Les critères sur lesquels sont basés l'opposition d'un Nord et d'un Sud qui légitime son action sont donc questionnés. En s'inscrivant désormais dans les pays dits du Nord, le commerce équitable ne relève plus seulement de l'étude des flux mondialisés mais appelle à une réflexion sur l'imaginaire spatial qu'il convoque. Que révèle la représentation graphique des figures de cet « habiter » le monde (Didelon Loiseau, 2010) ? L'objectif est de discuter un outil souvent débattu communément appelé carte mentale sur la question des territorialités du commerce équitable au regard des deux terrains de recherche; et ce dans un champ historiquement tourné vers d'autres espaces et des cadres d'analyses traditionnellement issus de l'économie. Après avoir présenté l'objet des territorialités du commerce équitable seront discutés à la fois la méthode des représentations graphiques ainsi que les enjeux de la recherche soulevés.

\section{Un attendu : exprimer des territorialités attachées au commerce équitable}

3 Dans la continuité des travaux sur la dimension conceptuelle du territoire initiés par G. Di Meo (1998), celui-ci est considéré dans sa logique processuelle et ses dynamiques d'acteurs. Le territoire est ainsi entendu comme « un espace approprié, avec sentiment ou conscience de son appropriation» (Brunet, 1992: 480), il est construit par les jeux d'acteurs (Vanier, 2009). Il n'est donc pas limité à une entité juridique ou institutionnelle qui est pourtant l'entrée du territoire dans la campagne" Fair Trade Towns", puisque les acteurs s'y projettent en termes de découpage de l'espace à la fois administratif (collectivité titrée) et fonctionnel (le titre comme des formes de pratiques, comme vecteur de valeurs), de gestion, d'aménagement, et s'y organisent en réseaux (Girard, 2012). Le projet' Fair Trade Towns' comme objet de la recherche s'insère dans ce cadrage théorique puisqu'il propose de nouvelles formes d'appropriation du territoire, et ce à travers les discours et les pratiques de l'ensemble des acteurs. Dans la lignée de la thèse de S. Girard (2012), la territorialité, qui renvoie à la dimension relationnelle du territoire à travers les intérêts, les conflits et stratégies d'acteurs (Vanier, 2009), et la territorialisation, construction territoriale en tant que processus évolutif et dynamique, sont distingués.

4 Les acteurs convoquent des imaginaires et des représentations du commerce équitable qui nourrissent : i) la territorialisation du secteur dans les politiques de développement durable; ii) les perceptions, ou les territorialités des acteurs concernés, à savoir des représentations individuelles même si elles sont en interactions avec le social. Les territorialités attachées au commerce équitable constituent une clé de lecture pour une meilleure compréhension des représentations, des imaginaires et des pratiques d'acteurs. 
Le recours à la représentation graphique par le dessin, comme outil, est utilisé conjointement à la conduite d'entretiens semi-dirigés. Le corpus est constitué de 48 entretiens et de 40 cartes mentales réalisés entre juillet 2013 et octobre 2014 avec les acteurs impliqués dans les" Fair Trade Towns", en France et en Belgique, sur la base des différents types d'acteurs identifiés dans le projet (Figure 1).

Figure 1. Présentation des structures rencontrées en entretien

\begin{tabular}{|c|c|c|c|c|c|}
\hline & $\begin{array}{l}\text { Acteurs du } \\
\text { commerce } \\
\text { équitable }\end{array}$ & Collectivités & $\begin{array}{l}\text { Entreprises, agences } \\
\text { et associations }\end{array}$ & $\begin{array}{l}\text { Acteurs } \\
\text { institutionnels }\end{array}$ & $\begin{array}{l}\text { Groupes } \\
\text { de } \\
\text { citoyens } \\
\text { et } \\
\text { citoyens }\end{array}$ \\
\hline France & \begin{tabular}{|l} 
Max Havelaar \\
Fédération \\
Artisans du \\
Monde \\
Plate-Forme \\
pour le \\
Commerce \\
Equitable \\
Fair (e) \\
Structures du \\
Collectif \\
Lyonnais des \\
Acteurs de \\
Commerce \\
Equitable
\end{tabular} & $\begin{array}{l}\text { Région Rhône } \\
\text { Alpes } \\
\text { Ville de Lyon }\end{array}$ & $\begin{array}{l}\text { Observatoire des } \\
\text { Achats Responsables } \\
\text { Association Régionale } \\
\text { de Développement } \\
\text { Rural Rhône-Alpes } \\
\text { Agencer De } \\
\text { l'Environnement et } \\
\text { de la Maîtrise de } \\
\text { l'Energie Agence } \\
\text { Française de } \\
\text { Normalisation } \\
\text { Structures labellisées } \\
\text { "Lyon Ville Equitable } \\
\text { et Durable " }\end{array}$ & $\begin{array}{ll}\text { Chambre } & \text { de } \\
\text { Commerce } & \text { et } \\
\text { d'Industrie de } \\
\text { Lyon } \\
\text { Réseau Urbact }\end{array}$ & -- \\
\hline Belgique & $\begin{array}{l}\text { Fair Trade } \\
\text { Advocacy } \\
\text { Office } \\
\text { Max Havelaar } \\
\text { Oxfam } \\
\text { Magasins du } \\
\text { Monde } \\
\text { Oxfam Liège } \\
\text { Miel Maya } \\
\text { Fédération } \\
\text { Belge pour le } \\
\text { commerce } \\
\text { équitable }\end{array}$ & $\begin{array}{l} \\
\text { Ville de } \\
\text { Bruxelles } \\
\text { Communes de } \\
\text { Bruxelles } \\
\text { Capitale } \\
\text { titrées ou } \\
\text { candidates }\end{array}$ & $\begin{array}{l}\text { Achats Verts } \\
\text { Biowallonie } \\
\text { «Quartiers durables » }\end{array}$ & $\begin{array}{lr}\text { Union } & \text { des } \\
\text { Communes } & \text { et } \\
\text { Villes } & \text { de } \\
\text { Wallonie } & \\
\text { Réseau Urbact }\end{array}$ & $\begin{array}{l}\text { Groupe } \\
\text { Berchem } \\
\text { Local Et } \\
\text { Durable }\end{array}$ \\
\hline \multicolumn{6}{|c|}{$\begin{array}{l}\text { Entretiens demandés restés sans réponse: Grand Lyon, Agence Française de Développement, Oxfam } \\
\text { Wereldwinkels, Commission Européenne, autres groupes «quartiers durables " de Bruxelles- } \\
\text { Capitale } \\
\text { Entretiens refusés: Parlement Bruxellois, Bruxelles-Environnement, réseau des consommateurs } \\
\text { responsables (Belgique) }\end{array}$} \\
\hline
\end{tabular}




\section{Le choix d'une méthode et ses questionnements}

5 L'utilisation de la représentation graphique représente un enjeu scientifique car elle interroge sur les moyens qu'elle a d'apporter des réponses à une question de recherche, cela en dépit d'un traitement et d'une analyse difficiles (Gumuchian, 1991 ; Bonin, 2004). La démarche méthodologique choisie est construite au regard de l'objet de recherche et ses clés de lecture, dans le cadre de la triangulation des données (Figure 2).

Figure 2. De l'intrigue à l'énigme, une méthode et des outils

Figure 2. De l'intrigue à l'énigme, une méthode et des outils

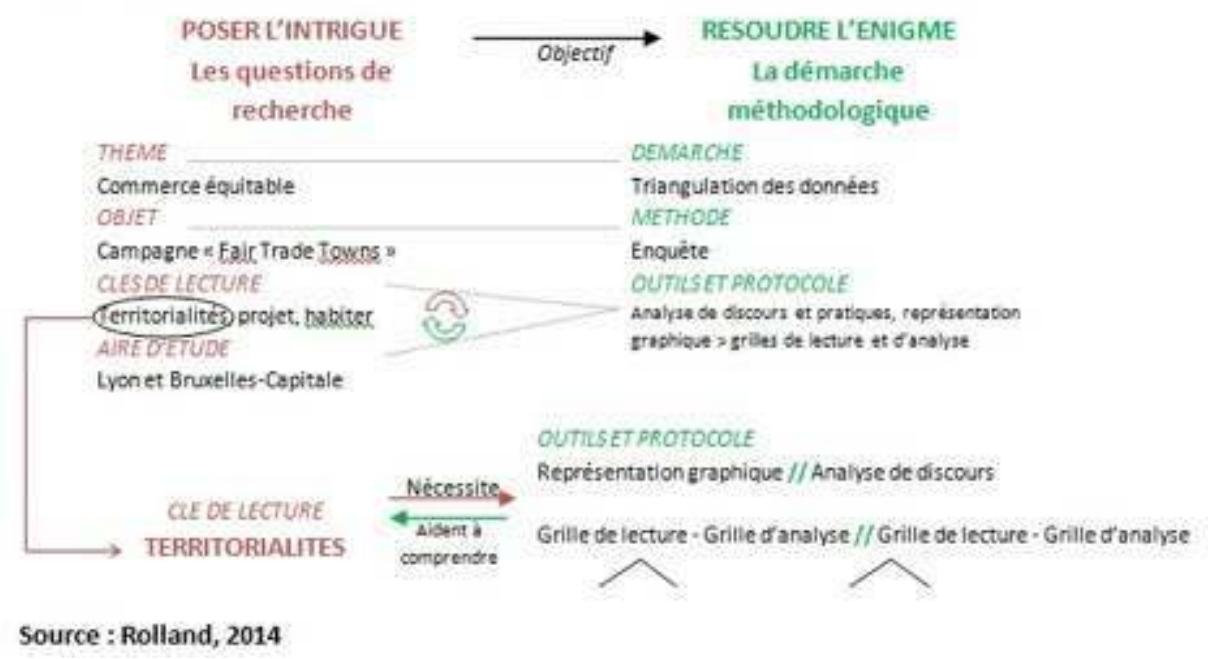

Source : Rolland, 2014

6 L'exercice est non contraint et réalisé sur papier blanc par les acteurs interrogés lors d'entretiens semi-directifs. Ces entretiens se structurent autour de trois thèmes-pivot inspirés des travaux de S. Lardon et V. Piveteau (2008) sur le territoire : points de vue et visions sur la campagne ; enjeux identifiés ; perspectives et aspirations. C'est à la toute fin de l'entrevue que la représentation graphique est proposée, en réponse à la consigne "Après avoir évoqué ces points, pouvez-vous maintenant représenter par le dessin ce qu'est pour vous un" Fair Trade Town" ?».

7 Les représentations sont rendues lisibles par les commentaires constants et/ou légendes de l'enquête durant le processus. A l'amont du terrain, et renforcé pendant son déroulement, un protocole de recherche est construit avec une grille de lecture des représentations (Figure 3). Les deux représentations graphiques choisies évoquent l'importance des liens et des échanges, ainsi que la structuration du phénomène étudié autour d'acteurs. Elles interrogent en ce sens l'outil en lui-même et sa lecture. 
Figure 3. Entre territoire institué et territoire ressenti : l'importance des liens et échanges qui structurent le phénomène
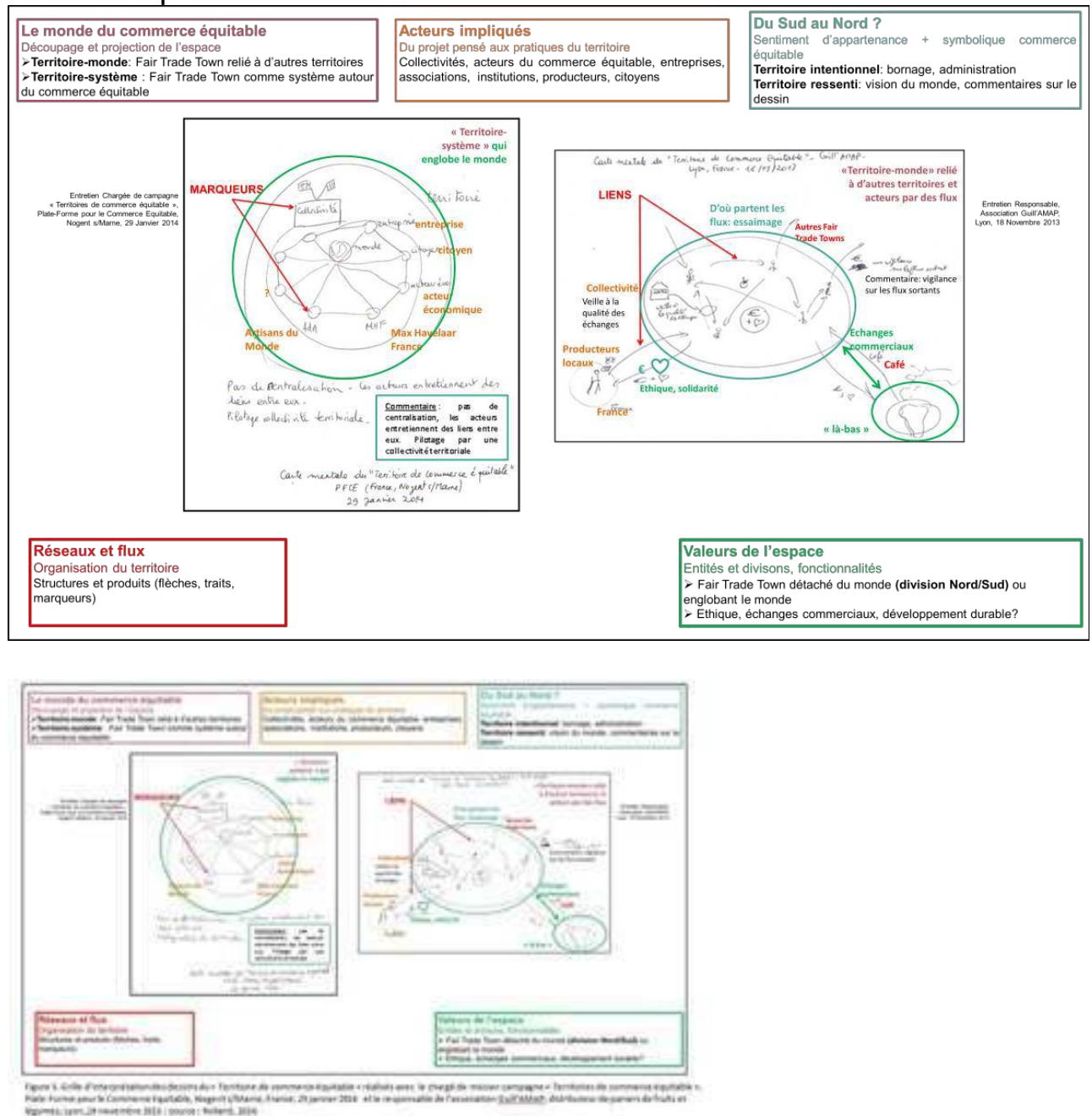

Grille d'interprétation des dessins du «Territoire de commerce équitable » réalisés avec le chargé de mission campagne "Territoires de commerce équitable ", Plate-Forme pour le Commerce Equitable, Nogent s/Marne, France, 29 janvier 2014 et le responsable de l'association Guill'AMAP, distributeur de paniers de fruits et légumes, Lyon, 18 novembre 2013;

source : Rolland, 2014

Deux lectures du territoire apparaissent. L'une exprime des valeurs, des idéaux, de l'ordre de l'intime, relatifs au commerce équitable à, l'autre illustre des éléments d'un territoire perçu dans sa dimension collective. Les dessins révèlent à la fois un territoire intentionnel (administration, drapeau, collectivité nommée et délimitée) et un territoire ressenti relevant des symboliques spatiales du commerce équitable (cœurs, échanges Nord Sud, liens entre acteurs).

Des ressemblances et des divergences apparaissent au prisme des positions des interrogés dans le système d'acteurs. Les similitudes tendent dans la qualification générale dü' Fair Trade Town" : son découpage, ses liens avec d'autres territoires ou le reste du monde ou non. L'accent est également ciblé sur les liens (humains, échanges de flux monétaires, de produits) entre les éléments qui structurent le phénomène étudié plus que sur leur emprise spatiale, tous recourant à des flèches, des traits reliant ces différents éléments dessinés. On peut ainsi plutôt parler d'un outil de schéma conceptuel (structuration du 
phénomène) que de carte mentale (emprise spatiale). Les divergences apparaissent qui relèvent de la signification du territoire attribuée (Figure 3) :

- Un «territoire-système» (Moine, 2007), où le" Fair Trade Town" englobe le monde avec le commerce équitable comme élément central ;

- Un « territoire-monde » (Didelon Loiseau, 2013), où le" Fair Trade Town" est relié à d'autres territoires et d'autres acteurs, par des flux de marchandises et des liens humains et culturels, à la fois du Nord au Sud et au Nord.

10 Par ailleurs, ces divergences sont aussi liées aux intérêts et stratégies dans le système d'acteurs. L'exemple du dessin issu de l'entretien avec la Plate-Forme pour le Commerce Equitable illustre le commerce équitable comme un élément fondateur du territoire, où les acteurs nationaux du commerce équitable estimés structurants du territoire sont notés et placés. Le dessin réalisé par le responsable d'une association de distribution de paniers de fruits et légumes labellisée par la Ville de Lyon convoque une vision centrée sur l'activité de la structure (agriculture locale) et d'autres territoires. La Ville de Lyon a créé son propre label «Lyon Ville Equitable et Durable » en 2010, suite à l'obtention de son titre "Territoire de commerce équitable " en 2009, labellisant à son tour des structures sur son territoire suivant d'autres critères de normalisation du développement durable. Enfin, des différences tiennent aussi des parcours des acteurs et de leur connaissance et expérience du commerce équitable, mais aussi de leur disposition à dessiner. Certains vont avoir une représentation plutôt schématique, avec des grands ensembles rectangulaires; d'autres vont essayer de cartographier en dessinant le monde, un pays, d'autres vont avoir recours à des symboles correspondant à des éléments du territoire (cercle, personnage schématisé pour un acteur). A cette étape, l'observation du dessin ainsi que l'enregistrement et/ou la prise de notes du commentaire réalisé par l'enquêté pendant l'exercice est essentielle en vue de l'analyse et de l'interprétation des dessins.

\section{Des enjeux d'interprétation des résultats et d'articulation entre théorie et résultats d'enquêtes}

11 Comment utiliser ces dessins ? Face à la diversité des approches, conjuguée à celle des territorialités du commerce équitable, une grille de lecture a recentré son application sur la problématique et les questions de recherche. Les enjeux sont de i) faire exprimer les territorialités des acteurs en complément des discours, illustrant la symbolique du projet et du territoire ; ii) faire apparaître les marqueurs spatiaux, les liens identifiés, parfois ambigus ou en contradiction avec les discours. Chaque dessin, et le commentaire l'expliquant, est « lu » en prenant en compte deux dimensions (Girard, 2012). La première dimension convoque l'identification ainsi que l'inscription dans l'espace du" Fair Trade Town ", c'est-à-dire son organisation, l'utilisation de figures spatiales (point, axe, surface délimitée) ainsi que l'établissement de relations spatiales entre ces objets ou avec l'observateur (flèches, cercles). La deuxième dimension concerne la signification de l'espace social, culturel ou symbolique du territoire, autrement dit l'attribution de fonction, d'usages, ou de valeurs attribuées à l'image organisée du " Fair Trade Town" , soit son sens, connoté par les espaces, les lieux traditionnels du commerce équitable, mais aussi par le registre discursif historique des acteurs du secteur sur le changement du système, une modification des règles internationales du commerce et des échanges. 
Le découpage de l'espace qui apparaît renvoie à des modèles et images attribués à ce que les acteurs identifient comme un Sud, face à un Nord, où chacun d'entre eux seraient des identités homogènes. Ils se réfèrent ainsi à un héritage des mouvements altermondialistes d'après-guerre qui ont fondé le commerce équitable. Malgré le décentrement du secteur opéré par la campagne les imaginaires du commerce équitable restent de même nature. Afin d'en comprendre les enjeux, une grille d'analyse éclaire les emboîtements d'échelles et de niveaux d'acteurs (Figure 4). Cet outil est conçu en parallèle de la grille d'analyse des entretiens de manière empirique. Dans la perspective de la triangulation des méthodes, la grille reprend les dimensions principales des catégories issues de l'analyse du discours des entretiens, à savoir les espaces identifiés et perçus dans la campagne "Fair Trade Towns", les acteurs structurants ainsi que le fonctionnement du territoire.

Figure 4. Grille d'analyse des dessins

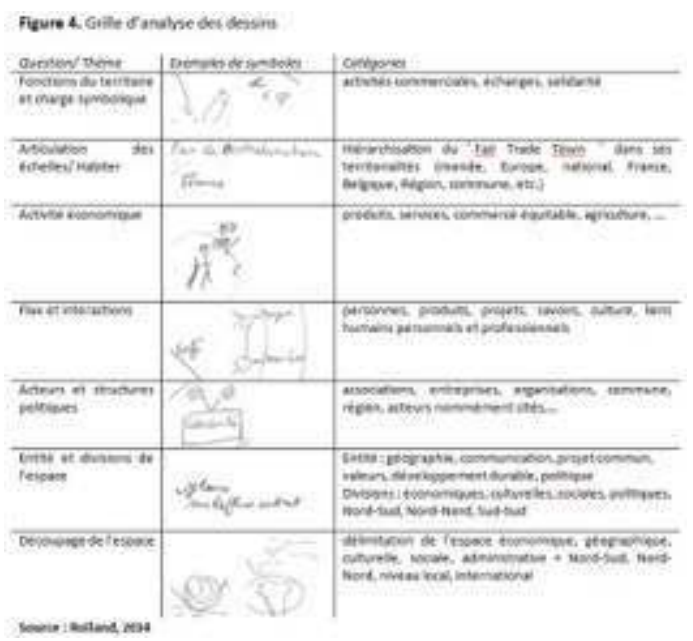

Source : Rolland, 2014

\section{Discussion sur la méthode au regard des études existantes}

L'état de l'art sur l'utilisation de la méthode des cartes mentales a été renforcé à la lecture de thèses de géographie et d'anthropologie urbaine récentes, et d'articles scientifiques sur le territoire (Avry, 2012 ; Bonnet et al, 2011 ; Debarbieux, Lardon, 2003 ; Faliès, 2013 ; Girard, 2012, Verguet, 2013). Si cette représentation de l'espace fait l'objet d'études depuis plusieurs décennies, à partir des travaux fondateurs sur la perception urbaine de K. Lynch (1960), elle ne fait pas l'objet d'une méthodologie consensuelle. L'interprétation de l'outil reste le problème majeur car elle est le produit de son contexte de réalisation et peut ainsi, en tant que produit construit, être saturé, ou non, d'informations. Elle dépend en effet des perceptions, "des pensées, des opinions et des sensibilités enfouies" (Bonnet et al, 2011) mais aussi de l'affect, de «non-représentations », ou de conditions de réalisation. Au regard de ces éléments, trois points de discussion sont soulevés.

La représentation graphique du territoire, au départ exploratoire en entretien, est devenue à mesure de la pratique de terrain un support majeur de la réflexion; et cela à travers les grilles de lecture et d'analyse propres au sujet de recherche. L'outil a participé 
à i) comprendre les représentations des acteurs sur le commerce équitable et sur sa mise en territoire convoquée par la campagne ; ii) révéler ou masquer des contradictions, des manques dans les discours; iii) dessiner un territoire dans la visée de construction de la notion au regard d'un objet de recherche nouveau dans la discipline géographique, le commerce équitable.

A travers les liens et les échanges représentés, la représentation graphique a renforcé le cadrage conceptuel de la recherche. Les notions d' « habiter » l'espace (Lussault, Pacquot, Younès, 2007), problématisée à l'échelle du territoire, et « d'habiter » le monde (Didelon Loiseau, 2010) éclairent la mise en territoire du commerce équitable. Ces entrées de lecture du commerce équitable issues de la catégorisation des dessins éclairent les emboîtements d'échelle en œuvre dans ce projet territorial.

Enfin, l'outil vise à analyser les échelles du commerce équitable convoquées dans cette campagne, angle nouveau des recherches qu'apporte une lecture géographique. Des interrogations en sont nées: parle-t-on d'habiter le monde, le territoire, la ville? Que veut dire cet «habiter»? La notion de territoire essaime-t-elle à travers cette campagne ? Les dessins produits ont replacé ce rapport au monde du commerce équitable au cœur de mon questionnement sur le terrain. Par ailleurs, ils font le lien entre ce travail et des recherches collectives menées par des chercheurs actuellement sur « Habiter le monde » (Université de Picardie Jules Verne).

\section{Conclusion}

Selon C. Yvroux et J.-P Bord (2011: 91), les représentations de l'espace sont «[...] envisagées à différentes échelles, souvent pour des espaces pratiqués et plus rarement pour ceux qui relèvent de l'« ailleurs », c'est-à-dire des espaces qui, pour un individu, ne sont appréhendés qu'à travers leurs dimensions idéelles». L'exemple des"Fair Trade Towns" illustre cet imaginaire géographique à l'aune du commerce équitable, qui fait appel à des espaces et des pratiques "inconnus» ou idéels. L'appréhension de la campagne est alors nourrie par des textes, des images, des pratiques quotidiennes et aussi par des représentations mentales d'un projet en construction. A travers cette recherche, la dichotomie Nord Sud est énoncée dans les discours comme fondement et aussi comme légitimation d'action du commerce équitable. Elle est spatialisée sur les dessins et abordée de manière plus fine dans le sens où les acteurs vont matérialiser des interactions et faire apparaître des schèmes qui complètent le discours. La méthode permet ainsi de préciser les différentes modalités de ce que peut être un territoire de commerce équitable. L'information qui en résulte doit être traitée et analysée au regard des autres outils méthodologiques utilisés, afin de mettre en évidence des stratégies, des intérêts ou encore des conflits d'acteurs. A travers l'exercice, l'acteur interrogé rappelle qu'il est un individu à plusieurs "casquettes ", qui va convoquer des territorialités relevant de sa position dans le système d'acteur au nom d'une structure, mais aussi de façon plus idéalisée en le faisant par son vécu, ses niveaux de connaissances et d'appropriation, et son affect. 


\section{BIBLIOGRAPHIE}

AVRY L. (2013), Analyser les conflits territoriaux par les représentations spatiales : une méthode cognitive par cartes mentales, Rennes, Université de Rennes2, $587 \mathrm{p}$. http://tel.archives-ouvertes.fr/docs/00/80/87/79/PDF/2012theseAvryL.pdf, consulté le 26 Février 2014.

BAILLY (1990), « Paysages et représentations », Mappemonde, 90/3, pp. 10-13

BONIN S. (2004), « Au-delà de la représentation, le paysage », Strates, 2004/11, 11p.

BONNET E. et al (2011), « Cartographier les représentations mentales du risque industriel. Illustration avec les populations de l'estuaire de la Seine - France », Congrès international de cartographie (ICC, 25Th), Paris, $11 \mathrm{p}$.

http://icaci.org/files/documents/ICC_proceedings/ICC2011/Oral\%20Presentations\%20PDF/A4-

Maps,\%20GIS,\%20image\%20processing\%20\&\%20hazards\%20and\%20disasters/CO-046.pdf, consulté le 10 Mars 2014.

BRUNET R. (1992), Les mots de la géographie. Dictionnaire critique, Paris, Reclus La documentation française, $518 \mathrm{p}$.

DI MEO G. (1998), Géographie sociale et territoire, Paris, Nathan Université, 317 p.

DIDELON LOISEAU C. (2010), « Une vision de l'Europe. Le tracé des chercheurs impliqués dans EPSON », Annales de Géographie, 673/3, pp. 211-228

http://www.armand-colin.com/download_pdf.php?idd=0\&cr=35\&idr=5\&idart=6426, consulté le 03 Mai 2014.

DIDELON LOISEAU C., (2013), Le Monde comme territoire, contribution à une approche renouvelée du Monde en géographie, Mémoire d'HDR, Volume 1, $321 \mathrm{p}$.

http://tel.archives-ouvertes.fr/docs/00/87/16/54/PDF/CDidelonLoiseau-HDR-Vol1.pdf, consulté le 15 Mai 2014.

FALIES C. (2013), Espaces ouverts et métropolisation entre Santiago du Chili et Valparaíso : produire, vivre et aménager les périphéries, Paris, Université Paris 1 Panthéon Sorbonne, $523 \mathrm{p}$. http://tel.archives-ouvertes.fr/docs/00/98/04/00/PDF/CFALIES_2013.pdf, consulté le 03 Mai 2014.

GIRARD S. (2012), La territorialisation de la politique de l'eau est-elle gage d'efficacité environnementale? Analyse diachronique de dispositifs de gestion des eaux dans la vallée de la Drôme (1970-2011), Lyon, Université de Lyon, 722 p. http://halshs.archives-ouvertes.fr/ docs/00/73/71/65/PDF/GIRARD_Sabine_2012_these_VD.pdf, consulté le 27 Février 2014.

GUMUCHIAN H. (1991), Représentation et aménagement du territoire, Paris, Anthropos, 135 p.

LARDON S. PIVETEAU V. (2008), « Méthodologie de diagnostic pour le projet de territoire : une approche par les modèles spatiaux », Géocarrefour, 80/2, pp. 75-90 http://geocarrefour.revues.org/980, consulté le 02 Février 2014.

LUSSAULT M. PACQUOT T. YOUNES C. (2007), Habiter, le propre de l'humain. Villes, territoires et philosophie, Paris, Armillaire, 384 p.

LYNCH K. (1960), L'image de la cité, Paris, Dunod, 222 p. 
MOINE A. (2007), Le territoire : comment observer un système complexe, Paris, L'Harmattan, $176 \mathrm{p}$.

SMITH A. (2011),

VANIER M. (2009), Territoires, territorialités, territorialisation. Controverses et perspectives, Rennes, Presses Universitaires de Rennes, 228 p.

VERGUET C. (2013), La fabrique ordinaire du patrimoine : étude de cas en milieu urbain, le quartier de la Libération à Nice, Nice, Université Sophia Antipolis, pp ?

YVROUD C., BORD J.P. (2011), « Représentations du conflit israélo-palestinien par la cartographie cognitive », CFC, 209, pp 91-102

http://www.lecfc.fr/new/articles/209-article-8.pdf, consulté le 27 Février 2014.

\section{RÉSUMÉS}

Un décentrement du secteur du commerce équitable est aujourd'hui perceptible vers les pays dits $\mathrm{du}$ Nord, autour de son intégration dans les politiques publiques de développement durable. La campagne européenne' Fair Trade Towns" en témoigne en prenant les formes d'un label décerné à des collectivités et d'un outil de communication. Ce projet questionne la dimension relationnelle du territoire par les jeux d'acteurs, ou les territorialités. A cet égard, la représentation graphique par le dessin est menée en entretien semi-directif avec les acteurs. Cet outil apparaît comme un support majeur de la lecture des territorialités, qui met en lumière des enjeux d'analyse dans le cadre de la démarche méthodologique.

A fair trade's growing shift towards the North appears with its integration into sustainable public policies. The European campaign "Fair Trade Towns" illustrates these new issues as his campaign is reading as one form of fair trade which entitle towns and also forms a communication's tool. "Fair Trade Towns" refers to the perception of the world by the actors, namely "subjective territories" encouraging fair trade. Schematic representations of those towns are realized during semi-structured interviews with the actors. This method can be a key to the interpretation of space and the perception of territories through fair trade. It also questions research and methodology stakes.

\section{INDEX}

Thèmes : Carnets de terrain

\section{AUTEUR}

\section{LISA ROLLAND}

Mosaïques, UMR7210 LAVUE

Université Paris Ouest Nanterre la Défense

UMR5600 EVS, Université de Lyon

Géographe

Lisa.Rolland[at]u-paris10.fr 